${ }^{1}$ Facultad de Medicina, Universidad Católica de la Santísima Concepción. Concepción, Chile.

${ }^{2}$ Programa Doctorado en Salud Mental, Departamento de Psiquiatría y Salud Mental, Facultad de Medicina, Universidad de Concepción. Concepción, Chile.

${ }^{3}$ Hospital Clínico Herminda Martín. Chillán, Chile. ${ }^{4}$ COSAM Chillán. Chillán, Chile. ${ }_{5}^{5}$ Program of Public Health, School of Health Sciences, Regis College, Weston, Massachusetts, USA. ${ }^{6}$ COSAM San Carlos. San Carlos, Chile.

${ }^{7}$ Dirección de Postgrado y Educación Continua, Universidad Diego Portales. Chile. aDoctor en Salud Mental. bPsicóloga(o).

'Doctor en Psicología.

dDoctor en Ciencias, Epidemiología.

'Magíster en Estadística Aplicada.

Fuente de apoyo financiero: El presente artículo no contó con apoyo financiero Los autores no declaran tener conflicto de intereses

Recibido el 29 de julio de 2017 , aceptado el 3 de diciembre de 2017.

Correspondencia a: Lilian Salvo G. Bulnes 473, Chillán. Isalvog@gmail.com

\section{Predictores de remisión del trastorno depresivo mayor en tratamiento en el nivel secundario de atención}

\author{
LILIAN SALVO ${ }^{1,2,3, a,}$, SANDRA SALDIVIA ${ }^{2, b, \mathrm{c}}$, CARLOS PARRA ${ }^{3,4}$, \\ MANUEL CIFUENTES ${ }^{5, \mathrm{~d}}$, CLAUDIO BUSTOS ${ }^{2, \mathrm{~b}, \mathrm{ce}, \mathrm{e}}$, PAOLA ACEVEDO ${ }^{3, \mathrm{~b}}$, \\ MARCELA DÍAZ ${ }^{3, \mathrm{~b}}$, MITZA ORMAZABAL ${ }^{4, \mathrm{~b}}$, IVONNE GUERRA ${ }^{4, \mathrm{~b}}$,

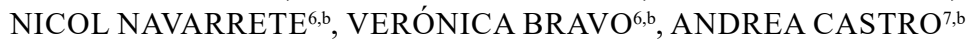

\section{Predictors of remission from major depressive disorder in secondary care}

Background: The knowledge of predictive factors in depression should help to deal with the disease. Aim: To assess potential predictors of remission of major depressive disorders (MDD) in secondary care and to propose a predictive model. Material and Methods: A 12 month follow-up study was conducted in a sample of 112 outpatients at three psychiatric care centers of Chile, with baseline and quarterly assessments. Demographic, psychosocial, clinical and treatment factors as potential predictors, were assessed. A clinical interview with the checklist of DSM-IV diagnostic criteria, the Hamilton Depression Scale and the List of Threatening Experiences and Multidimensional Scale of Perceived Social Support were applied. Results: The number of stressful events, perceived social support, baseline depression scores, melancholic features, time prior to beginning treatment at the secondary level and psychotherapeutic sessions were included in the model as predictors of remission. Sex, age, number of previous depressive episodes, psychiatric comorbidity and medical comorbidity were not significantly related with remission. Conclusions: This model allows to predict depression score at six months with 70\% of accuracy and the score at 12 months with $72 \%$ of accuracy.

(Rev Med Chile 2017; 145: 1514-1524)

Key words: Depression; Outpatients; Secondary Care.

\section{L} a depresión, entendida como enfermedad de etiología multifactorial y cuadro clínico heterogéneo, es considerada un problema de salud pública por su prevalencia relativamente alta, la discapacidad que causa, su frecuente comorbilidad y alto costo social ${ }^{1,2}$. Es un desafío disminuir su incidencia y carga de enfermedad. En esta línea cobra importancia el conocimiento de factores predictores del inicio y resultado.

Numerosos estudios evalúan predictores del resultado del trastorno depresivo mayor (TDM) en base a respuesta. Sin embargo, la evidencia demuestra que los pacientes con mejoría clínica, pero con síntomas residuales, presentan significativamente menor funcionamiento psicosocial y ocupacional, y mayor riesgo de recurrencia en comparación con remisión total ${ }^{3,4}$. De ahí la importancia de evaluar resultados en base a remisión.

Algunos estudios han demostrado que género femenino, edad mayor, menor nivel socioeconómico, desempleo y menor nivel educacional se asocian con riesgo de menor remisión o episodios depresivos (ED) de mayor duración ${ }^{5-10}$. Sin embargo, otros no evidencian tal relación ${ }^{11}$. Por otro lado, eventos estresantes, apoyo social y relaciones de pareja han mostrado baja asociación con remi- 
sión del ED. Se ha planteado que estas variables serían poco importantes en el curso de depresiones graves y recurrentes en contraste con el inicio y el resultado de ED leves, ${ }^{5,12,13}$. En adultos mayores, el apoyo social ha demostrado resultados contradictorios como predictor de remisión ${ }^{14,15}$. En lo clínico, de manera más consistente, empeoran el resultado: mayor severidad del $\mathrm{ED}^{16,17}$, mayor duración del episodio índice ${ }^{6,7}$, características psicóti$\operatorname{cas}^{18}$, comorbilidad psiquiátrica ${ }^{6,7,17}$, especialmente ansiedad $^{19-21}$, distimia ${ }^{6,15}$, trastorno por consumo de sustancias ${ }^{22}$ y trastorno de personalidad ${ }^{6,22-25}$. La comorbilidad médica también se ha asociado negativamente con remisión ${ }^{7,10,26,17,27,28}$.

La mayoría de la evidencia proviene de investigaciones efectuadas en la comunidad o en atención primaria, generalmente con diseños transversales o longitudinales con seguimiento corto. Menos estudios longitudinales se han realizado en TDM en el nivel especializado; es decir, en personas con episodios de mayor gravedad, comorbilidad, curso crónico o recurrente y/o resistencia a tratamiento; indagándose escasamente factores predictores de remisión del ED tratado en la práctica clínica real ${ }^{17}$.

En base a lo anterior, se realiza el presente estudio con el objeto de evaluar variables demográficas (sexo y edad), psicosociales (eventos estresantes y apoyo social percibido), clínicas (número de EDM previos, características melancólicas, comorbilidad psiquiátrica, comorbilidad médica, tiempo previo a tratamiento en el nivel secundario) y de tratamiento (intervenciones psicoterapéuticas), como potenciales predictores de remisión del TDM tratado en el nivel secundario de atención y proponer un modelo predictivo de remisión.

\section{Material y Método}

Estudio no experimental, longitudinal, con seguimiento durante doce meses, con evaluación basal y cada 3 meses.

\section{Participantes}

El universo lo constituyeron todas las personas con diagnóstico de TDM realizado por psiquiatra, con indicación de iniciar tratamiento en los tres centros de atención secundaria del Servicio de Salud de Nuble (SSÑ), entre el 01/08/2013 y 31/07/2014, de 15 y más años de edad. Los criterios de exclusión fueron trastorno bipolar, dependencia a sustancias en intoxicación o abstinencia, delirium, demencia, trastornos amnésicos, otros trastornos cognoscitivos, enfermedad médica y/o condición discapacitante que imposibilitara las evaluaciones y cambio de residencia fuera del SSÑ. A los que aceptaron participar se les efectuó entrevista clínica y check list DSM-IV para reconfirmar diagnóstico. Quienes fueron ratificados con TDM ingresaron al estudio, completándoseles evaluación basal e iniciando el seguimiento. El flujo de los participantes se presenta en la Figura 1. La muestra quedó constituida por 112 pacientes, $78,6 \%$ mujeres y $21,4 \%$ varones, de 15 a 79 años de edad, con promedio de 43,9 años (DE 15,3).

\section{Variable de resultado}

Remisión del EDM: definida según DSM-IV ${ }^{29}$ y evaluada por entrevista psiquiátrica y Escala de Depresión de Hamilton (Ham-D) ${ }^{30}$. Fue medida en evaluación basal (T0), a los 3 (T1), 6 (T2), 9 (T3) y 12 meses (T4). Según puntaje Ham-D, se consideró, sin remisión: $\geq 18$, remisión parcial: $8-17$, y remisión total: $\leq 7$ puntos $^{31}$.

\section{Variables predictoras}

Sexo. Edad.

Eventos estresantes negativos: definidos como eventos que requieren un reajuste en las actividades cotidianas de los individuos y que son percibidos como indeseables ${ }^{32,33}$. Fueron recogidos con el Listado de Eventos Estresantes (LEE), versión española ${ }^{34}$, en la evaluación T0, T2 y T4, consultándose por los 6 meses previos. Fue evaluado el número total de eventos y las categorías que resultaron más frecuentes.

Apoyo social percibido: conceptualizado como la capacidad de sentirse perteneciente a una trama de relaciones personales que proveen estimación y ayuda ${ }^{35}$. Medido por la Escala Multidimensional de Apoyo Social Percibido (EMASP) ${ }^{36}$, en la evaluación T0, T2 y T4 y operacionalizado por el puntaje total.

Número de ED previos: número de ED experimentados antes del episodio actual. Obtenidos en la entrevista psiquiátrica y complementados con registros de la historia clínica.

Características melancólicas: definidas según las especificaciones del DSM-IV. Se evaluó su presencia o ausencia por medio de checklist de criterios DSM-IV ${ }^{29}$.

Enfermedad psiquiátrica comórbida: $\mathrm{Co}-\mathrm{Ocu}-$ rrencia de dos o más trastornos psiquiátricos ${ }^{29,37}$. 


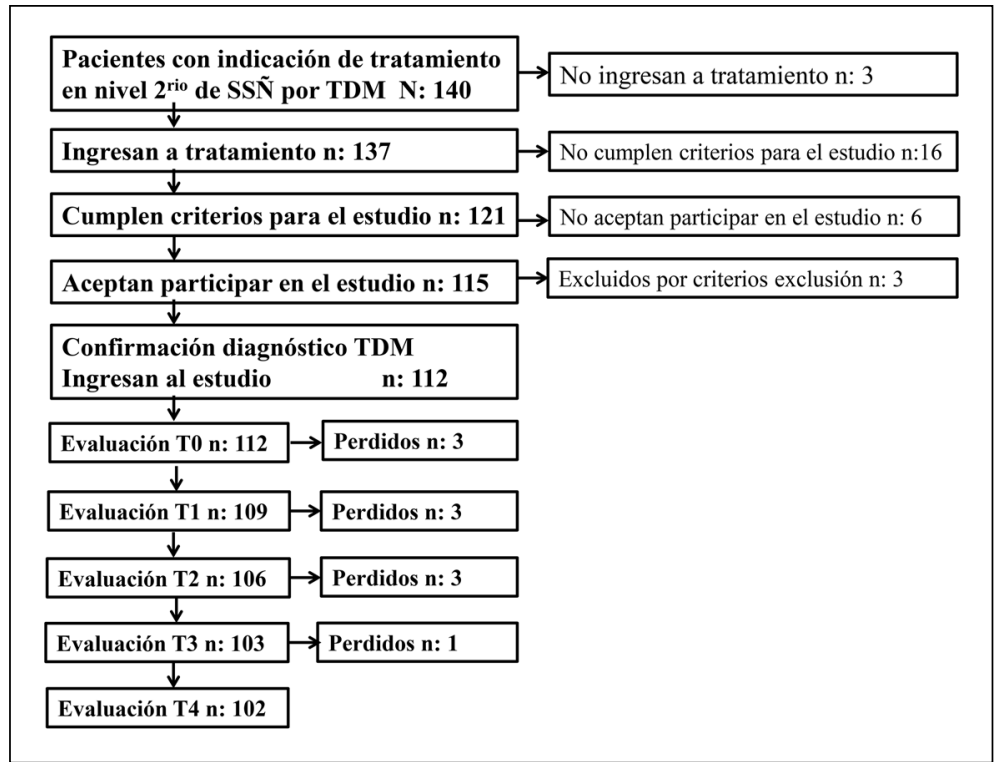

Figura 1. Flujo de participantes en estudio "Predictores de remisión del trastorno depresivo mayor en tratamiento en el nivel secundario de atención", del SSÑ, Chile. TDM= Trastorno Depresivo Mayor. SSÑ = Servicio de Salud de Ñuble.
El diagnóstico se efectuó a través de entrevista psiquiátrica según criterios DSM-IV ${ }^{29}$. Se evaluó su presencia o ausencia y las enfermedades que resultaron más frecuentes.

Enfermedad médica comórbida: co-ocurrencia de una o más enfermedades médicas y $\mathrm{TDM}^{29,37}$. Estos antecedentes fueron aportados por la(el) paciente y complementados con su historia clínica. Se analizó su presencia o ausencia y las enfermedades que resultaron más frecuentes.

Tiempo previo al inicio de tratamiento en el nivel secundario: tiempo transcurrido desde el inicio de tratamiento del EDM actual hasta el momento en que inicia tratamiento en el nivel secundario (meses). Esta información fue obtenida por psiquiatra en la evaluación basal más revisión de registros clínicos de referencia.

Intervenciones psicoterapéuticas: sesiones psicoterapéuticas individuales realizadas por psicólogo(a), registradas en historia clínica. Se consideró el número de sesiones. Para el análisis del modelo predictivo se consideró su efecto en la evaluación que correspondía y también en la siguiente (por efecto inmediato y a largo plazo).

\section{Instrumentos}

Entrevista psiquiátrica con checklist de criterios DSM-IV: entrevista clínica basada en los criterios diagnósticos del DSM-IV, de la Ameri- can Psychiatric Association ${ }^{29}$. Estos criterios son ampliamente usados en clínica e investigación.

Ham-D (versión 17 ítems): evalúa el perfil sintomático y gravedad de la depresión ${ }^{30}$. Extensamente usada en clínica e investigación. En ensayos clínicos el punto de corte más utilizado es $\leq 7$ : sin depresión; también empleado en $\mathrm{Chile}^{38}$. Su validez ha sido demostrada en nuestro medio ${ }^{39}$. En nuestro estudio, obtuvo un coeficiente alfa de Cronbach de 0,77.

LEE: diseñado por Bruga et $\mathrm{al}^{33}$ inicialmente para investigar factores de riesgo de depresión. Actualmente se recomienda y emplea en estudios de condiciones psiquiátricas, psicológicas y sociales. Consiste en un listado de 12 categorías de eventos estresantes negativos. Proporciona una puntuación total (entre 0 y 12 eventos) y/o presencia de cada categoría individual ${ }^{33}$. Se empleó la adaptación española ${ }^{34}$ que evidenció adecuados índices de fiabilidad y validez. A nivel internacional se usa ampliamente. Su validez se ha demostrado en nuestro país ${ }^{40}$.

EMASP: diseñada por Zimet et $a^{36}$ : compuesta por 12 ítems, recoge información del apoyo social percibido en: familia, amigos y otros significativos. Sus opciones de respuestas corresponden a una escala de acuerdo entre 1 y 4 puntos. El puntaje total puede oscilar entre 0 y 48 puntos. Posee excelente comportamiento psicométrico, en adultos 
y adolescentes ${ }^{41}$. Validada en Chile, ha obteniendo satisfactoria confiabilidad ${ }^{42} \mathrm{y}$ validez ${ }^{43}$. En la investigación actual, el Coeficiente alfa de Cronbach fue 0,87 .

\section{Procedimiento}

El estudio fue aprobado por los Comités de Ética de la Universidad de Concepción y SSÑ. Se coordinó la obtención de registros en cada centro. Identificado el ingreso, se contactó personalmente al paciente y se invitó a participar. Quienes aceptaron, firmaron el consentimiento informado para el estudio y para obtener información de su historia clínica. Las personas se incorporaron al estudio a medida que fueron ingresando a tratamiento. Las evaluaciones fueron realizadas en dependencias del establecimiento de salud donde asisten a atención. Todos fueron entrevistados individualmente por psiquiatra en la evaluación basal y por psicóloga en las evaluaciones siguientes.

\section{Análisis de los datos}

Los datos fueron analizados por medio del programa estadístico SPSS V21 y Mplus V 6.12. Las variables previamente determinadas (sexo, edad, número de eventos estresantes, apoyo social percibido, número de EDM previos, características melancólicas, comorbilidad psiquiátrica, comorbilidad médica, tiempo previo a tratamiento en el nivel secundario e intervenciones psicoterapéuticas) y aquellas que resultaron más frecuentes en el análisis descriptivo y significativas en análisis bivariado (enfermedad/lesión de familiar, muerte de amigo/familiar, problema grave con amigo/familiar, crisis económica grave, trastorno de personalidad cluster $\mathrm{B}$, cluster $\mathrm{C}$, con rasgos $\mathrm{B}-\mathrm{C}$ y dolor crónico), se sometieron al análisis multivariado, que se realizó a través de modelos lineales mixtos con mediciones repetidas. Las variables que aquí resultaron significativas en su relación con remisión (puntaje Ham-D), fueron incorporadas en el análisis para proponer un modelo predictivo de la remisión del EDM. Este último se efectuó por medio de análisis de curvas de crecimiento latente (CCL), considerándose los siguientes estadísticos: chi-cuadrado, grados de libertad, valor p, Comparative Fit Index (CFI), Root Mean Square Error of Approximation (RMSEA), Akaike information Criterion (AIC) y Bayesian Information Criterion (BIC). Se estableció nivel de significación de 0,05.

\section{Resultados}

Todos los pacientes fueron evaluados en la evaluación basal. El 91,1\% completó el seguimiento. Hubo una pérdida de $8,9 \%$ (10 pacientes). El seguimiento duró en promedio 11,8 meses (DE: 2,45), mínimo: 0 y máximo: 13,8 meses. En promedio, las evaluaciones fueron realizadas a 0,9 (DE: 0,85), 3,5 (DE: 0,67), 6,3 (DE: 0,53), 9,3 (DE: 0,68) y 12,4 (DE: 0,58) meses del ingreso al nivel secundario.

La descripción de las variables, el porcentaje de remisión y el tiempo para alcanzarla fue publicado

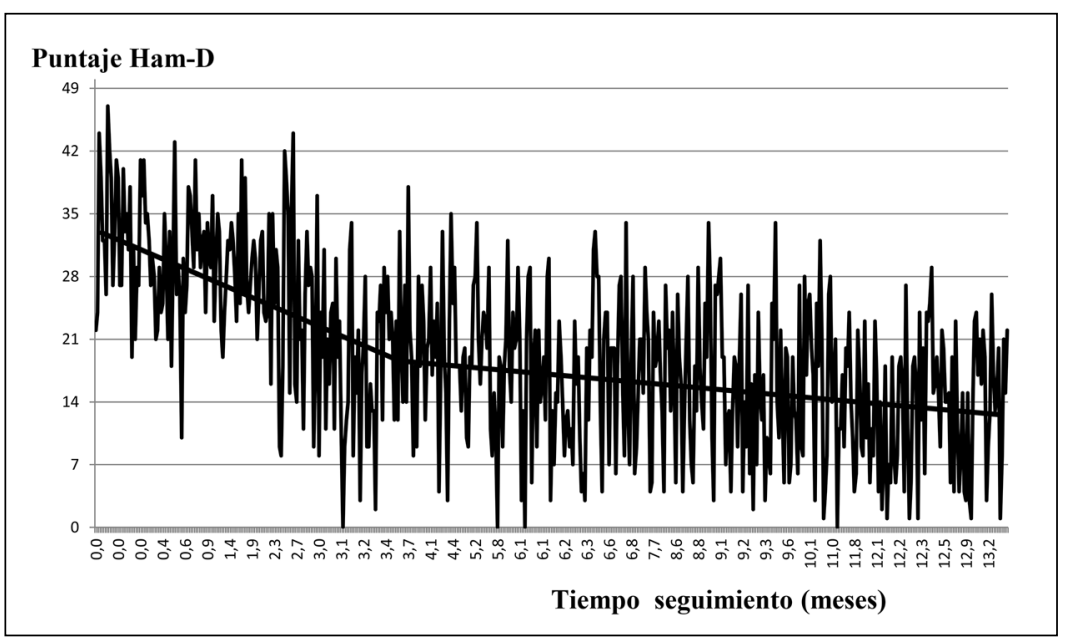

Figura 2. Curso de la remisión del EDM tratado en el nivel secundario del SSÑ, Chile (n: 112). Ham-D = Escala de Depresión de Hamilton. 
recientemente ${ }^{44}$. No hubo recurrencias durante el seguimiento. La Figura 2 muestra la evolución de los puntajes de depresión. La relación entre variables demográficas y psicosociales, clínicas y de tratamiento, con la remisión del EDM se presentan en las Tablas 1 y 2 , respectivamente.

En el análisis para proponer un modelo predictivo se modeló la trayectoria del descenso del puntaje Ham-D en forma posterior a la línea de base. El intercepto (I) se ubicó en la primera evaluación post ingreso (T1). La pendiente (S) refleja los cambios producidos en las tres evaluaciones posteriores.

El primer paso del análisis para el modelo predictivo consistió en ajustar el modelo de CCL al conjunto de datos disponibles. A continuación se evaluó la calidad de ajuste mediante estadísticos mencionados. Se encontró variabilidad individual

Tabla 1. Relación de variables demográficas y psicosociales con remisión del episodio depresivo mayor, en pacientes tratados en el nivel secundario del SSÑ (n: 112)

\begin{tabular}{|c|c|c|c|c|c|c|}
\hline \multirow[t]{2}{*}{ Variables } & \multirow[t]{2}{*}{ Coeficiente } & \multirow[t]{2}{*}{ DS } & \multirow[t]{2}{*}{$\mathbf{t}$} & \multirow[t]{2}{*}{$\mathbf{p}$} & \multicolumn{2}{|c|}{ IC $95 \%$} \\
\hline & & & & & Inferior & Superior \\
\hline \multicolumn{7}{|l|}{ Sexo } \\
\hline \multicolumn{7}{|l|}{ Puntaje depresión } \\
\hline Masculino & $-1,528$ & 1,41 & $-1,08$ & 0,280 & $-4,31$ & 1,25 \\
\hline Femenino & 0 & & & & & \\
\hline \multicolumn{7}{|l|}{ Pendiente } \\
\hline Masculino & 0,022 & 0,14 & 0,15 & 0,878 & $-0,26$ & 0,30 \\
\hline Femenino & 0 & & & & & \\
\hline \multicolumn{7}{|l|}{ Edad } \\
\hline Puntaje depresión & 0,081 & 0,04 & 2,11 & 0,035 & 0,01 & 0,16 \\
\hline Pendiente & 0,005 & 0,005 & 1,04 & 0,301 & $-0,005$ & 0,015 \\
\hline \multicolumn{7}{|l|}{$N^{\circ}$ eventos estresantes } \\
\hline Puntaje depresión & 0,541 & 0,21 & 2,54 & 0,011 & 0,12 & 0,96 \\
\hline Pendiente & 0,057 & 0,04 & 1,43 & 0,153 & $-0,02$ & 0,14 \\
\hline \multicolumn{7}{|c|}{ Enfermedad/lesión de familiar } \\
\hline Puntaje depresión & $\begin{array}{l}0,869^{a} \\
0^{b}\end{array}$ & 1,25 & 0,69 & 0,487 & $-1,59$ & 3,33 \\
\hline Pendiente & $\begin{array}{l}-0,342^{\mathrm{a}} \\
0^{\mathrm{b}}\end{array}$ & 0,18 & $-1,87$ & 0,062 & $-0,70$ & 0,017 \\
\hline \multicolumn{7}{|c|}{ Muerte amigo/familiar no padre/hijos } \\
\hline Puntaje depresión & $\begin{array}{l}-0,623^{\mathrm{a}} \\
0^{\mathrm{b}}\end{array}$ & 1,26 & $-0,49$ & 0,622 & $-3,11$ & 1,86 \\
\hline Pendiente & $\begin{array}{l}-0,104^{a} \\
0^{b}\end{array}$ & 0,18 & $-0,56$ & 0,576 & $-0,47$ & 0,26 \\
\hline \multicolumn{7}{|c|}{ Problema grave amigo/familiar } \\
\hline Puntaje depresión & $\begin{array}{l}-4,114^{a} \\
0^{b}\end{array}$ & 1,23 & $-3,34$ & 0,001 & $-6,54$ & $-1,69$ \\
\hline Pendiente & 0,148 & 0,19 & 0,78 & 0,434 & $-0,22$ & 0,52 \\
\hline \multicolumn{7}{|l|}{ Crisis económica grave } \\
\hline Puntaje depresión & $\begin{array}{l}-1,703^{\mathrm{a}} \\
0^{\mathrm{b}}\end{array}$ & 1,19 & $-1,43$ & 0,155 & $-4,05$ & 0,64 \\
\hline Pendiente & $\begin{array}{l}-0,341^{a} \\
0^{b}\end{array}$ & 0,18 & $-1,92$ & 0,055 & $-0,69$ & 0,01 \\
\hline \multicolumn{7}{|l|}{ Apoyo social } \\
\hline Puntaje depresión & $-0,102$ & 0,04 & $-2,29$ & 0,022 & $-0,19$ & $-0,02$ \\
\hline
\end{tabular}

Análisis multivariado. Todas las variables controladas por sexo y edad. Remisión medida por puntaje en Escala de Depresión de Hamilton. aausencia de la característica; ${ }^{\text {bp }}$ resencia de la característica. 
Tabla 2. Relación de variables clínicas y de tratamiento con remisión del episodio depresivo mayor, en pacientes tratados en el nivel secundario del SSÑ (n: 112)

\begin{tabular}{|c|c|c|c|c|c|c|}
\hline \multirow[t]{2}{*}{ Variables } & \multirow[t]{2}{*}{ Coeficiente } & \multirow[t]{2}{*}{ DS } & \multirow[t]{2}{*}{$\mathbf{t}$} & \multirow[t]{2}{*}{$\mathbf{p}$} & \multicolumn{2}{|c|}{ IC 95\% } \\
\hline & & & & & Inferior & Superior \\
\hline \multicolumn{7}{|c|}{ No Episodios depresivos previos } \\
\hline Puntaje depresión & 0,295 & 0,34 & 0,87 & 0,383 & $-0,37$ & 0,96 \\
\hline Pendiente & 0,003 & 0,03 & 0,10 & 0,921 & $-0,050$ & 0,055 \\
\hline \multicolumn{7}{|c|}{ Características melancólicas } \\
\hline Puntaje depresión & $-5,139^{a}$ & 0,91 & 5,66 & 0,000 & $-6,92$ & $-3,36$ \\
\hline Pendiente & $0,048^{a}$ & 0,17 & 0,28 & 0,781 & $-0,29$ & 0,39 \\
\hline \multicolumn{7}{|c|}{ Comorbilidad psiquiátrica } \\
\hline Puntaje depresión & $-1,879^{a}$ & 1,22 & 1,54 & 0,124 & $-4,28$ & 0,52 \\
\hline Pendiente & $0,177^{a}$ & 0,15 & 1,21 & 0,227 & $-0,47$ & 0,11 \\
\hline \multicolumn{7}{|c|}{ Trastorno de Personalidad B } \\
\hline Puntaje depresión & $-3,434^{a}$ & 1,59 & 2,16 & 0,031 & $-6,55$ & $-0,32$ \\
\hline Pendiente & $0,229^{a}$ & 0,22 & 1,03 & 0,305 & $-0,21$ & 0,67 \\
\hline \multicolumn{7}{|c|}{ Trastorno de Personalidad C } \\
\hline Puntaje depresión & $2,955^{\mathrm{a}}$ & 1,60 & 1,85 & 0,065 & $-0,18$ & 6,09 \\
\hline Pendiente & $-0,073^{a}$ & 0,23 & 0,32 & 0,750 & $-0,52$ & 0,37 \\
\hline \multicolumn{7}{|c|}{ Trastorno de Personalidad BC } \\
\hline Puntaje depresión & $-2,852^{a}$ & 1,62 & 1,76 & 0,079 & $-6,04$ & 0,33 \\
\hline Pendiente & $-0,116^{a}$ & 0,23 & 0,50 & 0,614 & $-0,57$ & 0,34 \\
\hline \multicolumn{7}{|l|}{ Comorbilidad médica } \\
\hline Puntaje depresión & $-1,412^{\mathrm{a}}$ & 1,18 & 1,20 & 0,232 & $-3,73$ & 0,91 \\
\hline Pendiente & $-0,144^{a}$ & 0,14 & 0,99 & 0,321 & $-0,43$ & 0,14 \\
\hline \multicolumn{7}{|l|}{ Dolor crónico } \\
\hline Puntaje depresión & $-3,529^{a}$ & 1,84 & 1,92 & 0,056 & $-7,15$ & 0,09 \\
\hline Pendiente & $0,022^{a}$ & 0,26 & 0,08 & 0,935 & $-0,49$ & 0,54 \\
\hline \multicolumn{7}{|c|}{ Tiempo previo a tratamiento niv2 } \\
\hline Puntaje depresión & 0,035 & 0,04 & 0,88 & 0,380 & $-0,04$ & 0,11 \\
\hline Pendiente & 0,008 & 0,00 & 2,06 & 0,040 & 0,0 & 0,02 \\
\hline \multicolumn{7}{|c|}{ Número sesiones psicoterapéuticas } \\
\hline Puntaje depresión & $-1,042$ & 0,63 & 1,65 & 0,101 & $-2,29$ & 0,20 \\
\hline Pendiente & 0,085 & 0,09 & 0,92 & 0,361 & $-0,10$ & 0,27 \\
\hline
\end{tabular}

Análisis multivariado. Todas las variables controladas por sexo y edad. Remisión medida por puntaje en Escala de Depresión de Hamilton. En todas las variables categóricas, la presencia de la característica tiene Coeficiente $=0$. a ausencia de la característica.

significativa en I y S (Tabla 3, modelo 1). El paso siguiente consistió en introducir las variables explicativas para predecir la variabilidad observada. Se evaluó: sexo, edad, número de eventos estresantes (en evaluación basal (T0), en evaluación T2 y T4), apoyo social percibido (en T0, T2 y T4), número de ED previos, características melancólicas, co- morbilidad psiquiátrica, trastorno de personalidad cluster B, comorbilidad médica, tiempo previo al inicio del tratamiento en el nivel secundario, puntaje basal de depresión y número de sesiones psicoterapéuticas. Posteriormente se analizaron distintos modelos (Tabla 3) hasta lograr el más parsimonioso, que se ajustara a los datos con el 
Tabla 3. Índices de ajustes para el modelo predictivo de remisión del episodio depresivo mayor, en pacientes tratados en el nivel secundario del SSÑ (n: 112)

\begin{tabular}{|c|c|c|c|c|c|c|c|}
\hline Modelo & $\chi^{2}$ & gl & $\mathbf{p}$ & CFI & RMSEA & AIC & BIC \\
\hline 1. Básico, sólo curva latente & 6,72 & 7 & 0,458 & 1 & 0 & 2.793 & 2.822 \\
\hline 2. Con todas las variables estables más curva & 26,08 & 27 & 0,514 & 1 & 0 & 2.806 & 2.889 \\
\hline $\begin{array}{l}\text { 3. Con todas las variables estables, variables que se modifican y } \\
\text { curva }\end{array}$ & 63,98 & 41 & 0,123 & 0,9 & 0,075 & 2.659 & 2.797 \\
\hline 4. Todas las variables menos trastorno de personalidad cluster B & 58,08 & 39 & 0,025 & 0,91 & 0,070 & 2.658 & 2.791 \\
\hline 5. Todas las variables menos sexo y trastorno personalidad B & 52,03 & 37 & 0,051 & 0,93 & 0,064 & 2.655 & 2.782 \\
\hline 6. Todas las variables menos edad y otras variables ya retiradas & 48,81 & 35 & 0,060 & 0,93 & 0,063 & 2.658 & 2.780 \\
\hline $\begin{array}{l}\text { 7. Todas las variables menos episodios depresivos previos y va- } \\
\text { riables ya retiradas }\end{array}$ & 41,49 & 33 & 0,147 & 0,96 & 0,051 & 2.655 & 2.772 \\
\hline $\begin{array}{l}\text { 8. Todas las variables menos comorbilidad médica y variables } \\
\text { retiradas }\end{array}$ & 34,32 & 31 & 0,312 & 0,98 & 0,033 & 2.653 & 2.765 \\
\hline $\begin{array}{l}\text { 9. Todas las variables menos comorbilidad psiquiátrica y variables } \\
\text { ya retiradas }\end{array}$ & 32,62 & 29 & 0,294 & 0,98 & 0,035 & 2.651 & 2.758 \\
\hline 10. Todas las variables menos apoyo social y variables ya retiradas & 23,84 & 23 & 0,413 & 0,99 & 0,019 & 2.674 & 2.765 \\
\hline $\begin{array}{l}\text { 11. Todas las variables con apoyo social menos eventos estresan- } \\
\text { tes y variables ya retiradas }\end{array}$ & 25,52 & 23 & 0,324 & 0,99 & 0,033 & 2.653 & 2.745 \\
\hline $\begin{array}{l}\text { 12. Todas las variables con apoyo social y eventos estresantes, } \\
\text { menos sesiones psicoterapéuticas y otras variables ya retiradas }\end{array}$ & 31,34 & 23 & 0,115 & 0,96 & 0,060 & 2.644 & 2.714 \\
\hline $\begin{array}{l}\text { 13. Todas las variables con apoyo social, eventos estresantes y } \\
\text { sesiones psicoterapéuticas a dos pasos, sin otras variables ya } \\
\text { retiradas }\end{array}$ & 35,31 & 32 & 0,315 & 0,98 & 0,032 & 2.643 & 2.731 \\
\hline $\begin{array}{l}\text { 14. Programa estadístico predice sesiones psicoterapéuticas, con } \\
\text { apoyo social y eventos estresantes, sin variables retiradas }\end{array}$ & 35,31 & 32 & 0,315 & 0,98 & 0,032 & 2.643 & 2.731 \\
\hline
\end{tabular}

Variables estables: sexo, edad, número de eventos estresantes basales, apoyo social percibido basal, puntaje de depresión (Ham) basal, número de episodios depresivos previos, características melancólicas, comorbilidad psiquiátrica, trastorno de personalidad cluster B, comorbilidad médica, tiempo previo al inicio del tratamiento en el nivel secundario. Variables que se modifican: número de eventos estresantes en la evaluación 2 y 4, apoyo social percibido en la evaluación 2 y 4 , y número de sesiones psicoterapéuticas

menor número de variables posibles. Se comenzó con un modelo que incluía todas las variables a evaluar (Tabla 3, modelo 3 y Figura 3), luego se retiraron progresivamente las que resultaban no significativas hasta obtener el modelo final, que de acuerdo con la calidad del ajuste $\left(\chi^{2}=35,31\right.$, $g l=32, p=0,315, C F I=0,98, R M S E A=0,032$, $A I C=2.643$ y $B I C=2.731)$ resultó ser el más parsimonioso (Tabla 3, modelo 13 y Figura 4). Éste incluyó: puntaje basal de depresión, características melancólicas, eventos estresantes, apoyo social percibido, tiempo previo al inicio de tratamiento en el nivel secundario y sesiones psicoterapéuticas (considerando su efecto en la evaluación correspondiente y en la siguiente evaluación).
Los coeficientes de determinación y niveles de significación para la capacidad explicativa del modelo se muestran en la Tabla 4 . El modelo propuesto permite predecir significativamente $58,6 \%$ del puntaje de depresión a los 3 meses, $69,6 \%$ a los 6 meses, $54,3 \%$ a los 9 meses y $72,4 \%$ a los 12 meses. En forma significativa puede predecir $24,3 \%$ del intercepto, pero no predice la pendiente de remisión.

\section{Discusión}

La gravedad basal de la depresión, las características melancólicas, el número de eventos 


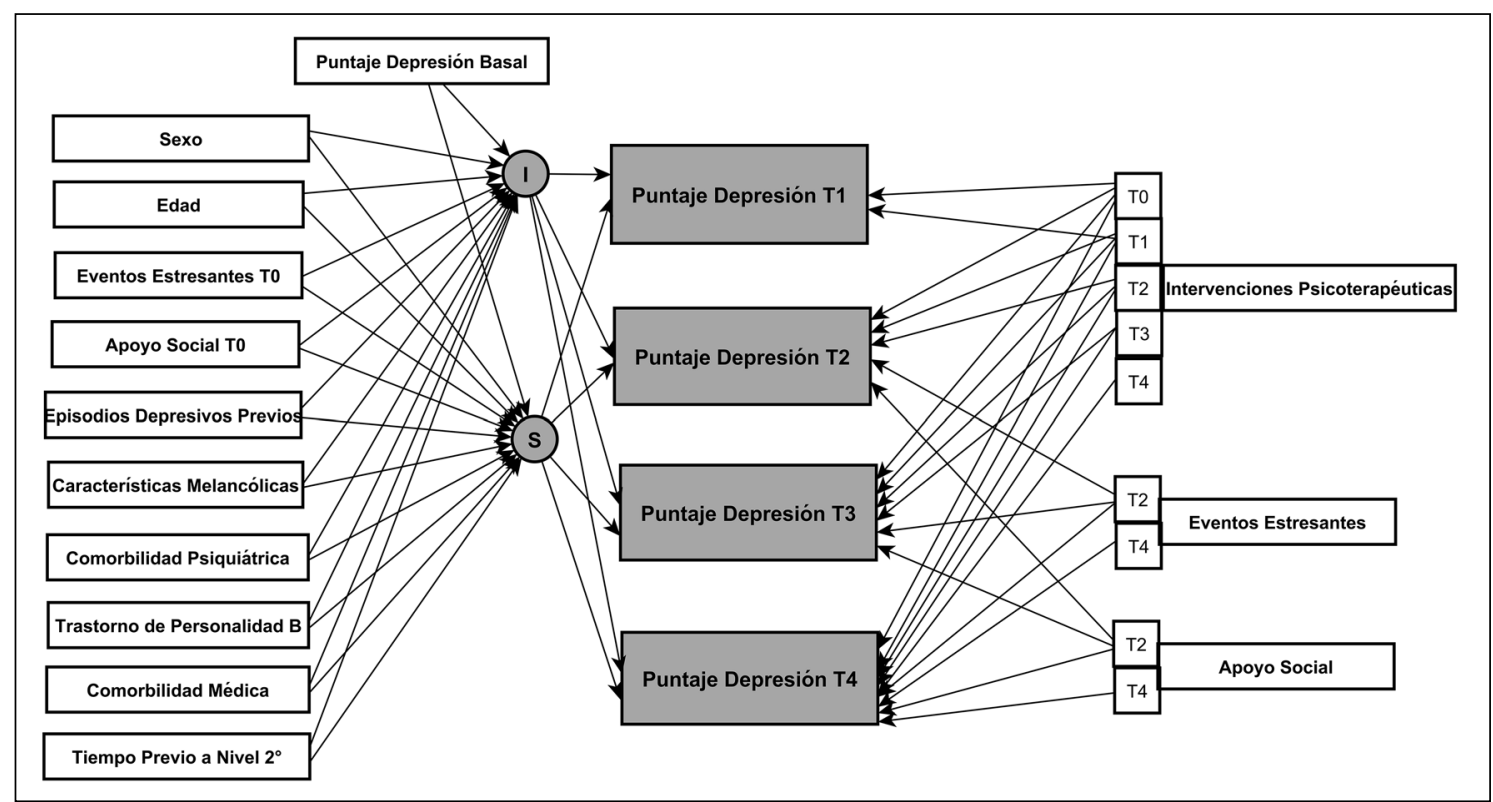

Figura 3. Ensayo inicial de modelo predictivo para la remisión del TDM tratado en el nivel secundario de atención del SSÑ con todas las variables en estudio (n: 112). I = Intercepto; $S=$ Pendiente; $T=$ Evaluación, $0=$ basal, $1=3$ meses, $2=6$ meses, $3=9$ meses, $4=12$ meses.

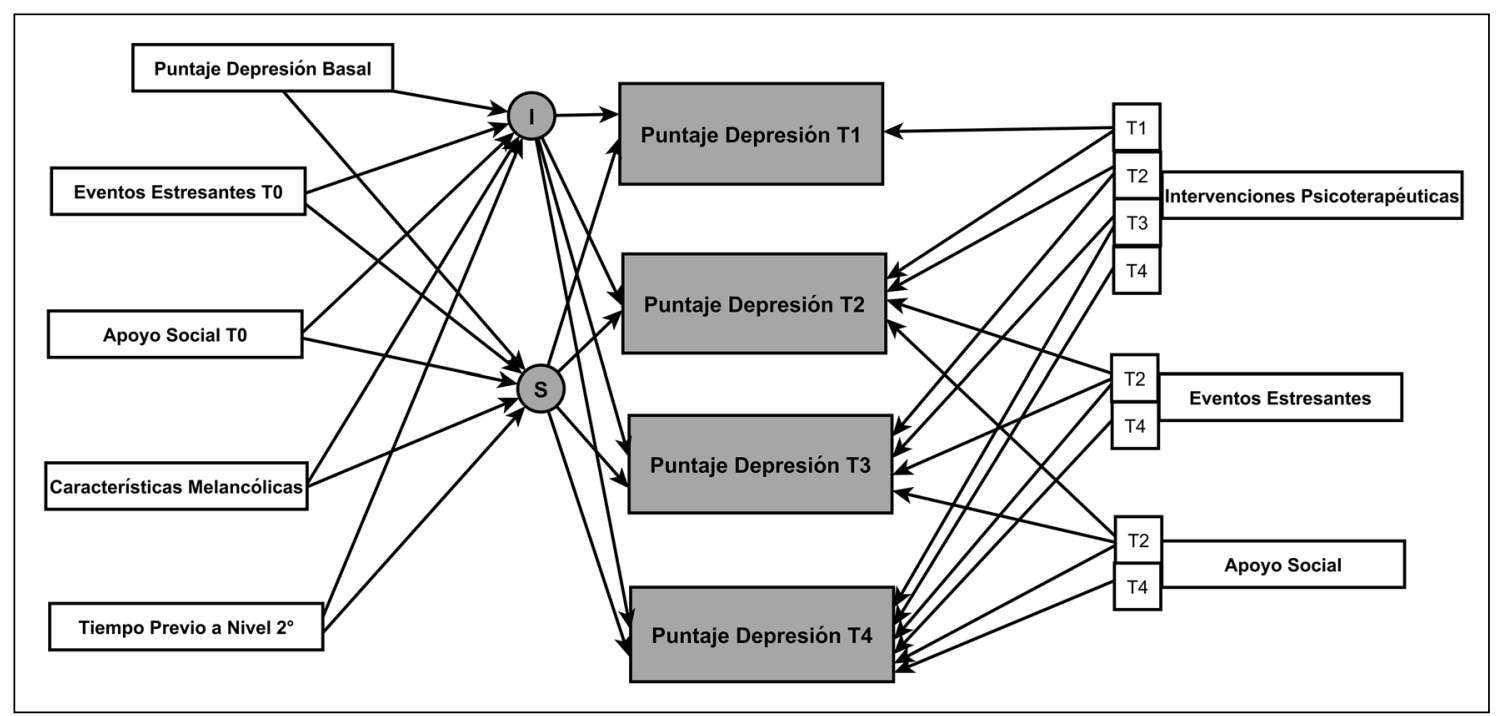

Figura 4. Modelo predictivo para la remisión del TDM tratado en el nivel secundario del SSÑ (n: 112 ). I = Intercepto; $\mathrm{S}=$ Pendiente; $T=$ Evaluación, $0=$ basal, $1=3$ meses, $2=6$ meses, $3=9$ meses, $4=12$ meses.

estresantes, el apoyo social percibido, el tiempo previo al tratamiento en el nivel secundario y las sesiones psicoterapéuticas predicen la remisión del EDM tratado en atención secundaria. El modelo propuesto, predice significativamente $70 \%$ del puntaje de depresión a los seis meses y $72 \%$ a los doce meses.

El predominio de mujeres en la muestra es concordante con otros estudios en población psiquiátrica ambulatoria ${ }^{6,7,17}$ y puede explicarse 


\section{Tabla 4. Coeficientes de determinación y niveles de significación para la capacidad explicativa del modelo predictivo de remisión del episodio depresivo mayor, en pacientes tratados en el nivel secundario del SSÑ (n: 112)}

\begin{tabular}{|lccc|}
\hline Variables & $\mathbf{r}^{\mathbf{2}}$ & DS & $\mathbf{p}$ \\
Variables observadas & & & \\
$\quad$ Ham1 & 0,586 & 0,09 & 0,000 \\
Ham2 & 0,696 & 0,06 & 0,000 \\
Ham3 & 0,543 & 0,06 & 0,000 \\
Ham4 & 0,724 & 0,09 & 0,000 \\
Variables latentes & & & \\
I & 0,243 & 0,10 & 0,012 \\
S & 0,543 & 0,53 & 0,307 \\
\hline
\end{tabular}

Ham1: Puntaje en Escala de Depresión de Hamilton, en evaluación T1 (3 meses). Ham2: Puntaje en evaluación T2 (6 meses). Ham3: Puntaje en evaluación T3 (9 meses). Ham4: Puntaje en evaluación T4 (12 meses). I: Intercepto. S: Pendiente.

por la mayor prevalencia del TDM en el sexo femenino.

Este estudio reafirma la mayor gravedad basal de la depresión, el mayor número de eventos estresantes y el menor apoyo social como predictores de menor remisión del EDM, ya publicado por otros autores ${ }^{6,14,17}$. Proporciona nueva información respecto a las características melancólicas, no estudiadas como predictores previamente. Como en un estudio finlandés ${ }^{6}$, encontramos que la duración del tiempo previo al nivel secundario es un predictor significativo. Las investigaciones y guías clínicas se han centrado más en el tipo de psicoterapias efectivas que en intensidad de las intervenciones psicoterapéuticas, pero en Chile generalmente la atención pública no cuenta con profesionales especializados en técnicas específicas por lo que estos resultados proporcionan evidencia para replantearse dichas intervenciones.

No encontramos publicaciones con modelos predictivos para remisión del ED grave para comparar el porcentaje de predicción. Un estudio que evaluó síntomas depresivos en esclerosis múltiple encontró que el modelo explicó $25 \%$ de los síntomas iniciales de depresión ${ }^{45}$. Otros autores han usado CCL para indagar trayectorias de respuesta a tratamiento en depresión ${ }^{46}$.

De acuerdo con los resultados, se hace evidente la necesidad de: 1) Educar e intervenir a la comunidad para consulta precoz en depresión o en factores de riesgo y capacitar líderes comunitarios en detección; lo que ayudaría a diagnóstico y tratamiento más precoz; 2) Capacitar continuamente a equipos de atención primaria, mejorar la red y su referencia interna, con el objeto de calificar características, gravedad, tipo de tratamiento y disminuir los tiempos de derivación al nivel secundario; 3) Corregir protocolo GES y su cobertura, incluyendo derivación al nivel secundario de las depresiones con síntomas melancólicos y depresiones graves aunque no presenten intento de suicidio y/o síntomas psicóticos; 4) Realizar protocolos para diagnóstico y tratamiento que respeten normativas ministeriales, complementados con estrategias según características locales; 5) Capacitar a psicólogas(os) en tipo y regularidad de las intervenciones psicoterapéuticas; 6) Organización de grupos comunitarios, articulados con la red de salud, con acción intersectorial de una política de estado, lo cual podría tener efecto en algunos tipos de eventos estresantes, en apoyo social y en rehabilitación; y 7) Continuar investigando para nueva información y para evaluar las estrategias implementadas.

Este estudio cuenta con limitaciones: período de seguimiento, diseño observacional, no permite atribuciones causales y no es representativo de la realidad nacional. Es deseable un mayor tamaño muestral.

Sus fortalezas radican en ser el primer estudio con mayor seguimiento que evalúa resultados de depresión de mayor gravedad en Chile, realizado en ambiente real de sistemas público, incluyendo pacientes habitualmente excluidos en investigaciones. Usó entrevistas psiquiátricas e instrumentos estandarizados empleados universalmente y análisis multivariados modernos. Tuvo bajo porcentaje de pérdidas y permanecieron pacientes que abandonaron tratamiento.

\section{Referencias}

1. Fried ME, Nesse RM. Depression is not a consistent syndrome: An investigation of unique symptom patterns in the STAR ${ }^{\star}$ D study. J Affect Disord 2014; 172: 96-102.

2. Marcus M, Yasamy MT, Van Ommeren M, Chisholm D, Saxena S. Depression. A global public health concern. OMS. 2012. Disponible en: www.who.int/mental_ health/management/depression/who_paper_depression_wfmh_2012.pdf [consultado el 17 de marzo de 2017]. 
3. Hardeveld F, Spijker J, De Graaf R, Nolen WA, Beekman AT. Recurrence of major depressive disorder and its predictors in the general population: results from The Netherlands Mental Health Survey and Incidence Study (NEMESIS). Psychol Med 2013; 43: 39-48.

4. Romera I, Pérez V, Menchón JM, Delgado-Cohen H, Polavieja P, Gilaberte I. Social and occupational functioning impairment in patients in partial versus complete remission of a major depressive disorder episode. A six-month prospective epidemiological study. Eur Psychiatry 2010; 25 (1): 58-65.

5. Stegenga BT, Geerlings MI, Torres-González F, Xavier M, Svab I, Penninx BW, et al. Risk factors for onset of multiple or long major depressive episodes versus single and short episodes. Soc Psychiatry Psychiatr Epidemiol 2013; 48 (7): 1067-75.

6. Holma KM, Holma IA, Melartin TK, Rytsala HJ, Isometsa ET. Long-term outcome of major depressive disorder in psychiatric patients is variable. J Clin Psychiatry 2008; 69: 196-205.

7. Trivedi MH, Rush AJ, Wisniewski SR, Nierenberg AA, Warden D, Ritz L, et al. Evaluation of outcomes with citalopram for depression using measurement-based care in $\mathrm{STAR}^{\star} \mathrm{D}$ : implications for clinical practice. Am J Psychiatry 2006; 163 (1): 28-40.

8. Azar AR, Chopra MP, Cho LY, Coakley E, Rudolph JL. Remission in major depression: results from a geriatric primary care population. Int J Geriatr Psychiatry 2011; 26 (1): 48-55.

9. Hung CI, Wang SJ, Liu CY, Hsu SC, Yang CH. Comorbidities and factors related to discontinuation of pharmacotherapy among outpatients with major depressive disorder. Compr Psychiatry 2011; 52 (4): 370-7.

10. Ciudad A, Álvarez E, Roca M, Baca E, Caballero L, García de Polavieja P, et al. Early response and remission as predictors of a good outcome of a major depressive episode at 12-month follow-up. J Clin Psychiatry 2012; 73 (2): 185-91.

11. Mulder RT, Joyce PR, Frampton CM, Luty SE, Sullivan PF. Six months of treatment for depression: outcome and predictors of the course of illness. Am J Psychiatry 2006; 163 (1): 95-100.

12. Paykel ES, Cooper Z, Ramana R, Hayhurst H. Life events, social support and marital relationships in the outcome of severe depression. Psychol Med 1996; 26: 121-33.

13. Bock C, Bukh JD, Vinberg M, Gether U, Kessing LV. Do stressful life events predict medical treatment outcome in first episode of depression?. Soc Psychiatry Psychiatr Epidemiol 2009; 44 (9): 752-60.

14. Bosworth HB, Hays JC, George LK, Steffens DC. Psy- chosocial and clinical predictors of unipolar depression outcome in older adults. Int J Geriatr Psychiatry 2002; 17 (3): 238-46.

15. Hybels CF, Blazer DG, Steffens DC. Predictors of partial remission in older patients treated for major depression: the role of comorbid dysthymia. Am J Geriatr Psychiatry 2005; 13 (8): 713-21.

16. Katzelnick DJ, Duffy FF, Chung H, Regier DA, Rae DS, Trivedi MH. Depression outcomes in psychiatric clinical practice: using a self-rated measure of depression severity. Psychiatr Serv 2011; 62 (8): 929-35.

17. Novick D, Hong J, Montgomery W, Dueñas H, Gado M, Haro JM. Predictors of remission in the treatment of major depressive disorder: real-world evidence from a 6-month prospective observational study. Neuropsychiatr Dis Treat 2015; 11: 197-205.

18. Craig TJ, Grossman S, Bromet EJ, Fochtmann LJ, Carlson GA. Medication use patterns and two-year outcome in first-admission patients with major depressive disorder with psychotic features. Compr Psychiatry 2007; 48 (6): 497-503.

19. Kim JM, Kim SW, Stewart R, Kim SY, Yoon JS, Jung SW, et al. Predictors of 12 -week remission in a nationwide cohort of people with depressive disorders: the CRESCEND study. Hum Psychopharmacol 2011; 26: 41-50.

20. Coryell W, Fiedorowicz JG, Solomon D, Leon AC, Rice JP, Keller MB. Effects of anxiety on the long-term course of depressive disorders. Br J Psychiatry 2012; 200 (3): 210-5.

21. Fava M, Rush AJ, Alpert JE, Balasubramani GK, Wisniewski SR, Carmin CN, et al. Difference in treatment outcome in outpatients with anxious versus no anxious depression: a STAR ${ }^{\star}$ D report. Am J Psychiatry 2008; 165 (3): 342-51.

22. Davis LL, Wisniewski SR, Howland RH, Trivedi MH, Husain MM, Fava M, et al. Does comorbid substance use disorder impair recovery from major depression with SSRI treatment? An analysis of the $\mathrm{STAR}^{\star} \mathrm{D}$ level one treatment outcomes. Drug Alcohol Depend 2010; 107 (2-3): 161-70.

23. Grilo CM, Stout RL, Markowitz JC, Sanislow CA, Ansell EB, Skodol AE, et al. Personality disorders predict relapse after remission from an episode of major depressive disorder: a 6-year prospective study. J Clin Psychiatry 2010; 71: 1629-35.

24. Asano T, Baba H, Kawano R, Takei H, Maeshima H, Takahashi $\mathrm{Y}$, et al. Temperament and character as predictors of recurrence in remitted patients with major depression: A 4-year prospective follow-up study. Psychiatry Res. 2015; 225 (3): 322-5. 
25. Skodol AE, Grilo CM, Keyes KM, Geier T, Grant BF, Hasin DS. Relationship of personality disorders to the course of major depressive disorder in a nationally representative sample. Am J Psychiatry 2011; 168 (3): 257-64.

26. Azar AR, Chopra MP, Cho LY, Coakley E, Rudolph JL. Remission in major depression: results from a geriatric primary care population. Int J Geriatr Psychiatry 2011; 26 (1): 48-55.

27. Novick D, Montgomery W, Bertsch J, Peng X, Brugnoli $\mathrm{R}$, Haro JM. Impact of painful physical symptoms on depression outcomes in elderly Asian patients. Int Psychogeriatr 2014; 4: 1-8.

28. Borza T, Engedal K, Bergh S, Benth JS, Selbæk G. The course of depression in late life as measured by the Montgomery and Asberg Depression Rating Scale in an observational study of hospitalized patients. BMC Psychiatry 2015; 15: 191.

29. Asociación Americana de Psiquiatría: Manual Diagnóstico y Estadístico de los Trastornos Mentales (DSM-IVTR). Barcelona: Masson; 2009.

30. Hamilton M. A rating scale for depression. J Neurol Neurosurg Psychiatry 1960; 23: 56-62.

31. Paykel ES, Ramana R, Cooper Z, Hayhurst H, Kerr J, Barocka A. Residual symptoms after partial remission: an important outcome in depression. Psychol Med 1995; 25: $1171-80$.

32. Monroe SM. Modern approaches to conceptualizing and measuring human life stress. Annu Rev Clin Psychol 2008; 4: 33-52.

33. Brugha TS, Cragg D. The List of Threatening Experiences: the reliability and validity of a brief life events questionnaire. Acta Psychiatr Scand 1990; 82: 77-81.

34. Motrico E, Moreno-Küstner B, De Dios Luna J, Torres-González F, King M, Nazareth I, et al. Psychometric properties of the List of Threatening Experiences - LTE and its association with psychosocial factors and mental disorders according to different scoring methods. J Affect Disord 2013; 150(3): 931-40.

35. Uchino B, Cacioppo J, Kiecolt-Glasser J. The relationship between social support and physiological processes: a review with emphasis on underlying mechanism and implications for health. Psychol Bull 1996; 119: 488-531.
36. Zimet G, Dahlem N, Zimet S, Farley G. Multidimensional Scale of Perceived Social Support. J Pers Assess 1988; 52 (1): 30-41.

37. Valderas J, Starfield B, Sibbaald B, Salisbury C, Roland M. Defining Comorbidity: implications for understanding health and health services. Ann Fam Med 2009; 7: 357-63.

38. Retamal P, Florenzano R, Vacarezza A, Chamorro X, Armijo I, Leighton C, et al. Evaluación del tratamiento de depresión severa: un estudio en un centro especializado de referencia: Rev Latinoamericana Psiquiatría 2006; 6: 18-24.

39. Ojeda C, Silva H, Cabrera J, Tchimino C, De la Carrera C, Molina A. Validez interna y concurrente de una escala breve autoadministrada de siete ítems para la depresión, evaluada en relación a los inventarios de Beck y Hamilton. Estudio preliminar. Rev Chil Neuro-Psiquiat 2003; 41 (1):31-8.

40. Vásquez J, Panadero S, Rincón P. Stressful Life Events and Suicidal Behaviour in Countries with Different Development Levels: Nicaragua, El Salvador, Chile and Spain. J. Community Appl Soc Psychol 2010; 20: 288-98.

41. Dahlem N, Zimet G, Walker R. The multidimensional Scale of Perceived Social Support: a confirmation study. J Clin Psychol 1991; 47 (6): 756-61.

42. Ortiz M, Baeza MJ. Propiedades psicométricas de una escala para medir apoyo social percibido en pacientes chilenos con diabetes tipo 2. Universitas Psychologica 2011; 10 (1): 189-96.

43. Vivaldi F, Barra E. Bienestar Psicológico, Apoyo Social Percibido y Percepción de Salud en Adultos Mayores. Terapia Psicológica 2012; 30(2): 23-9.

44. Salvo L, Saldivia S, Parra C, Rodríguez R, Cifuentes M, Acevedo $\mathrm{P}$, et al. Características clínicas del trastorno depresivo mayor en tratamiento en el nivel secundario de atención. Rev Med Chile 2017; 145: 335-43.

45. Beal C, Stuifbergen A, Sands D, Brown A. Depression in multiple sclerosis: A longitudinal analysis. Arch Psychiatr Nurs 2007; 21 (4): 181-91.

46. Muthén B, Asparouhov T. Growth Modeling with Non-Ignorable Dropout: Alternative analyses of the $\mathrm{STAR}^{\star} \mathrm{D}$ Antidepressant Trial. Psychol Methods 2011; 16 (1): 17-33. 